\title{
Associations Between Intraindividual Variability in Sleep and Daily Positive Affect
}

\author{
Fei Ying $^{1}\left(\mathbb{D} \cdot\right.$ Jin H. Wen ${ }^{1} \cdot$ Patrick Klaiber ${ }^{1} \cdot$ Anita DeLongis $^{1} \cdot$ Danica C. Slavish ${ }^{2} \cdot$ Nancy L. Sin $^{1}$
}

Received: 15 March 2021 / Accepted: 16 September 2021/ Published online: 9 November 2021

(c) The Society for Affective Science 2021

\begin{abstract}
Recent research highlights a variety of negative outcomes associated with intraindividual variability in positive affect (PA) and in sleep. Thus, this study examined the associations of variability in multiple dimensions of sleep (quality, duration, wake after sleep onset, bedtime, rise-time) with mean and variability in PA. For 7 days, morning and evening surveys were collected online from two separate samples: community-based adults $(N=911)$ and university students $(N=322)$. Regression analyses revealed that across both samples, people with more variable sleep quality exhibited greater fluctuations in PA throughout the week and, surprisingly, higher levels of PA on average. In the community sample only, individuals with more variable sleep duration had lower and more variable PA. Findings suggest that fluctuations in sleep quality and duration are linked with daily PA outcomes, which is important to consider as technological advances and modern demands make inadequate and irregular sleep increasingly common.
\end{abstract}

Keywords Affect variability $\cdot$ Sleep health $\cdot$ Daily diary $\cdot$ COVID-19 $\cdot$ Affect dynamics

Positive affect (PA) is often defined as the experience of pleasurable emotions such as happiness, excitement, and contentment (Lyubomirsky et al., 2005). A large body of evidence suggests that PA has unique benefits for health (Pressman et al., 2019), with higher trait PA being linked to lower inflammation (Steptoe et al., 2005), reduced risk of cardiovascular disease (Boehm \& Kubzansky, 2012), and lower mortality risk (Chida \& Steptoe, 2008). Sleep has been increasingly recognized as a key factor influencing both emotional functioning and health (Kahn et al., 2013; Zohar et al., 2005). Although several dozen studies have linked better sleep to higher PA (Ong et al., 2017), the role of variability and level of sleep in PA dynamics is unknown. Given that fluctuations in PA and sleep have separately been linked to worse health outcomes (Bei et al., 2016; Ong \& Steptoe, 2020), and that technological advances and modern social/

Handling Editor: Aric Prather

Nancy L. Sin

nsin@psych.ubc.ca

1 Department of Psychology, University of British Columbia, 2136 West Mall, Vancouver, BC V6T 1Z4, Canada

2 Department of Psychology, University of North Texas, Denton, TX, USA work demands have increased the prevalence of irregular sleep (Gradisar et al., 2013), it is important to further investigate the relationship between intraindividual variability in sleep and PA. The current study examined variability in sleep as a predictor of mean levels and variability in PA during the COVID-19 pandemic, a period with heightened stress (Zheng et al., 2021) and changes in sleep patterns (Rezaei \& Grandner, 2021).

\section{Variability in Sleep and PA}

Intraindividual variability in affect represents the extent to which affect fluctuates within an individual over a specific period of time (most commonly quantified by intraindividual standard deviation [iSD]; Ong \& Ram, 2017). Researchers have speculated that rapid fluctuations between extreme highs and lows in affect across a short period of time can be maladaptive for physical and mental health (Gruber et al., 2013). These fluctuations might reflect environmental demands that evoke rapid shifts in affect or possibly poorer affect regulation skills (Ong \& Ram, 2017). Indeed, multiple cross-sectional studies have shown that people with greater variability in PA tend to have higher inflammation (Jones et al., 2020), less-favorable cortisol profiles (Human 
et al., 2015), worse immune response (Jenkins et al., 2018), more chronic health conditions (Chan et al., 2016), and more psychological distress (Gruber et al., 2013; Hardy \& Segerstrom, 2017). Recently, data from the English Longitudinal Study of Aging showed that instability in PA (measured as the mean of squared successive differences) predicted allcause mortality across 12 years, independent of mean levels of PA (Ong \& Steptoe, 2020).

The existing literature on sleep-wake patterns has focused largely on average levels of sleep indices (such as quality, duration, and efficiency) rather than night-to-night fluctuations (Bei et al., 2016). However, given that individuals might experience short, fragmented sleep one night, and consequently sleep longer and more soundly the next night (Mezick et al., 2009), intraindividual variability in sleep may reveal information that is not captured by betweenperson comparisons of averages alone. Indeed, Mezick et al. (2009) found that individuals who experienced more stressors over the course of 9 days had greater variability in actigraphy-measured sleep duration and fragmentation, independent of person-means for sleep parameters. In a population-representative adult sample and in nurses, those with greater variability in self-reported or actigraphic sleep duration had higher risks for inflammation, pain, depression, and medical conditions (Slavish et al., 2019; Slavish et al., 2020, 2021). In their systematic review, Bei et al. (2016) observed that people who had greater variability in one or more sleep-wake parameters (e.g., duration, sleep onset latency, bedtime, or rise-time) also reported more stress, worse physical health, depressive symptoms, and overall poorer sleep.

\section{The Relationship Between Sleep and PA}

The current study focused on sleep as a predictor of PA mean and variability. Although the relationship between sleep and PA is often conceptualized as bidirectional, ambulatory studies that directly test both directions of association have found more consistent support for sleep as a predictor of next-day affect than vice versa (e.g., Bouwmans et al., 2017; Sin et al., 2017, 2020). Neurobiological research has found that rapid eye movement (REM) sleep may be particularly important for promoting appropriate emotional reactivity (Goldstein \& Walker, 2014). Regarding emotion dynamics, our study draws on the conceptual framework proposed by Palmer and Alfano (2017), which utilizes the process model of emotion regulation (Gross, 1998) to understand the influence of sleep on emotion regulatory processes. According to this model, the neurobiological and behavioral effects of inadequate sleep can impact multiple aspects of emotion generation and regulation, including situation selection, situation modification, attentional deployment, appraisals, and response modulation (Palmer \& Alfano, 2017). For instance, Palmer and
Alfano (2017) explain that poor sleep can diminish motivation to seek out or fully engage in rewarding activities (e.g., social interactions) that would otherwise promote PA. In addition to shaping one's environment, disrupted sleep can impair attentional strategies normally used to regulate one's affective responses (Palmer \& Alfano, 2017). For example, sleep deprivation reduces accuracy in recognizing positive but not negative facial expressions (Maccari et al., 2014). Thus, we expect that more variable sleep (reflecting poorer or disrupted sleep patterns) will be associated with lower mean PA and greater PA variability, perhaps reflecting diminished situational selection or emotion regulation.

Previous research has shown that sleep is related to nextday affect and vice versa. For example, in a sample of 1,025 community-based adults using the same dataset as the current study, we found that nights of better, longer, or more efficient sleep - compared to one's usual level of sleep-predicted better affective well-being, fewer physical symptoms, and lower odds of stressor occurrence on the following day (Wen et al., in press). However, separate from within-person processes, a growing body of research has shown that between-person differences in affect variability are related to sleep dimensions. For instance, above and beyond traitlike levels of PA, Ong et al. (2013) found that individuals with greater PA fluctuations in response to daily events had lower actigraphic sleep efficiency, compared to those with less event-related affect. In addition, people with greater variability in momentary PA also had shorter self-reported sleep duration and greater morning tiredness (Leger et al., 2019). Of note, Leger et al. (2019) also observed associations between iSD in negative affect (NA) and average sleep quality and morning tiredness, but these associations were no longer significant after controlling for mean NA. Additional experimental evidence has demonstrated that a single night of sleep continuity disruption attenuates next-day state PA, inhibition of pain by PA, and attentional bias to positive stimuli (Finan et al., 2017). Considering ambulatory, experimental, and longitudinal research demonstrating the unique associations of sleep indices with PA (Ong et al., 2017), the current study focused on PA, while controlling for NA.

\section{Current Study}

The objective of the current study was to examine the relationship between variability in sleep and PA. Two samples, community-dwelling adults $(N=911)$ and university students $(N=322)$, reported their previous night's sleep and current-day affect in twice-daily surveys for 1 week during the COVID-19 pandemic. We examined characteristics of sleep that have been most clearly associated with better mental and physical well-being, based on Buysse's SATED (satisfaction, alertness, timing, efficiency, and duration) model of sleep health (Buysse, 2014). Specifically, we examined 
self-reported sleep quality as an indicator of satisfaction, bedtime and rise-time for timing, wake after sleep onset (WASO) for efficiency, and sleep duration; unfortunately, the current study did not capture alertness/sleepiness. Based on previous evidence that variable sleep patterns are associated with poorer psychological health outcomes (Bei et al., 2016), we hypothesized that greater intraindividual variability in sleep quality, bedtime, rise-time, WASO, and sleep duration would be associated with lower mean PA, as well as greater day-to-day variability in PA. A better understanding of the role of sleep variability in PA dynamics could help guide efforts to improve both sleep and well-being. This has important public health significance given the prevalence of societal-level changes in sleep patterns (Rezaei \& Grandner, 2021) and declines in psychological well-being during the COVID-19 pandemic (Prati \& Mancini, 2021; Robinson et al., 2021; Zheng et al., 2021).

\section{Methods}

\section{Design and Participants}

The data used were collected from the online "Coping with the COVID-19 Outbreak" study from March to August 2020. Data, code, and materials are available on the Open Science Framework website at https://osf.io/wq5xh/?view_only= 062ae85dafcf4fafb16658128a1a5191. For seven consecutive days, participants completed morning surveys about their prior night's sleep and evening surveys about current-day affect. Participants were emailed links to the Qualtrics surveys at 7 A.M. and 7 P.M. local time.

Two samples were recruited for this study, and they were examined separately in parallel sets of analyses. The first was a sample of community-dwelling adults aged 18-91 $(N=1,135)$ living in Canada and the USA. They were recruited without monetary incentives via advertisements in various news outlets, community organizations, and on social media. To ensure that each participant had enough days of observation to capture day-to-day variability in affect and sleep, we excluded participants $(n=182)$ who completed fewer than 4 of 7 survey days. On average, participants from the community sample completed $4.96(S D=1.49)$ of 7 study days. An additional 42 participants were excluded for missing key variables: age $(n=2)$, sex $(n=12)$, education $(n=1)$, race $(n=26)$, and sleep duration variability (unable to calculate due to participant reporting error; $n=1$ ). The final analytic size for the community sample was 911 participants.

The second sample consisted of undergraduate students $(N=386)$ at a large Canadian university. They received psychology course credit for their participation in the study, which was available to students during March and April
2020. Of these, 55 participants were excluded for completing fewer than 4 of 7 survey days, and an additional 9 participants were excluded for missing key variables: sex $(n=1)$, race $(n=5)$, negative affect variability $(n=2)$, and sleep duration variability $(n=1)$. On average, student participants completed $5.04(S D=1.38)$ of 7 survey days. The final analytic size for the student sample was 322 participants. All participants in both samples provided written informed consent, and procedures were approved by the university research ethics review board.

\section{Measures}

\section{Daily Affect}

Daily affect was assessed in the evening survey with items adapted from the National Study of Daily Experiences (Sin et al., 2020). Based on previous research on the intraindividual factor structure of emotions in daily life (Charles et al., 2019), we used items that tapped into core components of PA that vary day-to-day, namely emotional experiences representing cheerfulness (happy), contentment (calm, satisfied), agency (confident, proud, enthusiastic, full of life), and interpersonally-oriented states (like you belong, close to others). Of note, the pattern of findings was similar with or without the two interpersonally-oriented PA items.

Every evening, participants reported the extent to which they had experienced 9 positively-valenced emotions for PA (enthusiastic, happy, satisfied, confident, calm, like you belong, close to others, proud, full of life) and 7 negativelyvalenced emotions for NA that day (anxious, sad, angry, frustrated, disgusted, lonely, ashamed; Sin, Klaiber, Wen, et al., 2021; Sin, Rush, et al., 2021). Ratings were made using a visual analogue scale that ranged from 0 (not at all) to 100 (extremely). We calculated between-person reliability $\left(R_{\mathrm{KF}}\right)$ and within-person reliability $\left(R_{\mathrm{C}}\right)$ using methods described by Cranford et al. (2006). The affect scales had good reliability between-persons $\left(R_{\mathrm{KF}}=0.98\right.$ for NA, 0.99 for PA) and within-persons $\left(R_{\mathrm{C}}=0.74\right.$ for NA, 0.85 for PA).

\section{Sleep Measures}

Participants completed the Consensus Sleep Diary in the morning survey (Carney et al., 2012). In line with Buysse's (2014) multidimensional model of sleep health, we examined five sleep measures: bedtime, rise-time, WASO reported in minutes, subjective sleep quality assessed on a visual analogue scale $(0=$ very poor, $100=$ very good $)$, and sleep duration calculated by subtracting the time spent awake in bed (WASO plus sleep onset latency) from the total time in bed (interval between bedtime and rise-time). 


\section{Data Analysis}

Data analyses were conducted in R, version 4.0.2 (R Core Team, 2020). Following previous research (Eid \& Diener, 1999), we calculated intraindividual variability as the iSD for each participant's reports of affect and sleep across all their days of observation. Intraindividual or within-person standard deviation is the most widely used measure of variability for both PA (Houben et al., 2015; Ong \& Ram, 2017) and sleep (Bei et al., 2016). Due to extreme outliers, WASO variability was winsorized to a maximum of 2 standard deviations above the mean. We analyzed the community and student samples separately. For each sample, we first conducted bivariate correlations among sleep and affect variables. Next, linear regression models were used to evaluate each sleep variability index (bedtime, rise-time, sleep duration, WASO, sleep quality) as predictors of mean PA and PA variability.

All models included the following covariates: age, sex (male vs. female), education level (less than 4-year college degree vs. 4-year college degree and above), self-reported chronic illness status ("I have a chronic medical condition such as heart disease, lung disease, or diabetes"), and proportion of weekend days (calculated as the number of surveys completed on the weekend divided by the total number of surveys completed). Based on differences in the racial composition of the two samples (see Table 1), we controlled for white versus non-white race in the community sample, whereas race was coded as white, Asian, or other race in the student sample. To determine whether the relationship between sleep variability and PA held above and beyond person-mean values, we controlled for person-means of the sleep measures. We also controlled for NA person-mean and variability to determine whether the relationship between sleep and PA was unique from NA. In the models predicting PA variability, we further controlled for mean PA, while in the models predicting mean PA, we further controlled for PA variability. All continuous predictors and covariates were centered at the grand mean.

\section{Results}

\section{Descriptives and Correlations}

Participant characteristics for the community and student samples are shown in Table 1. Both samples were predominantly female. The community sample was largely white (89.0\%), whereas over half of the student sample was Asian $(52.8 \%)$. As expected, the student sample was younger and most did not yet hold a 4-year college degree. On average, the student sample had later and more variable bed- and risetimes, as well as longer and more variable sleep duration.
Table 1 Descriptive characteristics

\begin{tabular}{|c|c|c|}
\hline \multirow[t]{2}{*}{ Variable } & \multicolumn{2}{|l|}{ Mean $(S D)$ or $N(\%)$} \\
\hline & Community sample & Student sample \\
\hline \multicolumn{3}{|l|}{ Participant characteristics } \\
\hline Age, years & $46.52(16.02)$ & $21.20(3.89)$ \\
\hline Female sex & $801(87.9 \%)$ & $246(76.40 \%)$ \\
\hline White race & $811(89.0 \%)$ & $92(28.6 \%)$ \\
\hline Asian & - & $170(52.8 \%)$ \\
\hline Other & - & $60(18.6 \%)$ \\
\hline $\begin{array}{l}\text { 4-year college degree and } \\
\text { above }\end{array}$ & $633(69.48 \%)$ & $43(13.4 \%)$ \\
\hline Chronic disease & $203(22.3 \%)$ & $13(4.0 \%)$ \\
\hline Positive affect & $48.17(18.78)$ & $43.31(17.37)$ \\
\hline Negative affect & $24.90(15.70)$ & $26.07(14.88)$ \\
\hline Bedtime, hour & $11: 20 \mathrm{PM}(1.41)$ & 1:23AM (1.72) \\
\hline Rise-time, hour & $8: 10 \mathrm{AM}(1.50)$ & 10:14AM (1.73) \\
\hline Sleep duration, hours & 7 h $25 \min (0.96)$ & 7 h $48 \min (1.15)$ \\
\hline WASO, minutes & $24.02(26.11)$ & $8.25(14.21)$ \\
\hline Sleep quality & $58.64(17.38)$ & $59.56(16.88)$ \\
\hline \multicolumn{3}{|l|}{ Intraindividual variability } \\
\hline $\begin{array}{l}\text { Positive affect variability, } \\
\text { iSD }\end{array}$ & $20.41(5.96)$ & $20.25(6.16)$ \\
\hline $\begin{array}{l}\text { Negative affect variability, } \\
\text { iSD }\end{array}$ & $22.34(8.44)$ & $21.66(8.02)$ \\
\hline Bedtime variability, iSD & $0.77(0.71)$ & $1.34(1.21)$ \\
\hline Rise-time variability, iSD & $1.15(1.20)$ & $1.63(1.50)$ \\
\hline $\begin{array}{l}\text { Sleep duration variability, } \\
\text { iSD }\end{array}$ & $1.12(0.63)$ & $1.43(0.86)$ \\
\hline WASO variability, iSD & $22.62(20.45)$ & $9.51(13.38)$ \\
\hline Sleep quality variability, iSD & $18.51(8.46)$ & $17.37(9.12)$ \\
\hline
\end{tabular}

$N=911$ persons and 5,242 days for community sample. $N=322$ persons and 1,828 days for student sample. Affect and sleep quality were rated on scales from 0 to 100

The community sample had greater and more variable WASO. Both samples were similar in their average levels and variability for PA, NA, and sleep quality.

Bivariate between-person correlations among sleep and affect variables in the community sample are shown in Supplementary Table S1. People with higher mean PA tended to have less variability in PA, bedtime, rise-time, WASO, and sleep duration. Higher mean PA was also correlated with means levels of sleep: earlier bedtime and rise-time, lower WASO, longer duration, and higher quality. Greater PA variability was significantly correlated with greater variability in sleep duration and in sleep quality, as well as later mean bedtime. Most sleep variability measures were significantly correlated with one another, except there were no correlations between variability in rise-time and in sleep quality nor between variability in bedtime and in WASO. 
Bivariate between-person correlations among sleep and affect variables in the student sample are shown in Supplementary Table S2. In students, mean PA was generally not correlated with the sleep measures, although higher mean PA was correlated with higher mean sleep quality. Furthermore, there was no correlation between mean PA and PA variability in the student sample. Greater PA variability was correlated with higher mean and larger variability in sleep quality, but no other sleep measures. Finally, similar to the community sample, most sleep variability measures were correlated with one another, except there was no correlation between rise-time variability and sleep quality variability, nor bedtime variability and WASO variability.

\section{Multiple Regression Analyses}

In all regression models in the community sample (Tables 2 and 3, Supplementary Tables S3, S4, and S5), there was an inverse relationship between mean PA and mean NA, and between mean PA and NA variability. Additionally, people with lower mean NA and greater NA variability both reported more variable PA. Similar relationships were observed in the student sample, except mean NA was no longer a significant predictor of mean PA after controlling for NA variability. In the community sample only, older age was associated with higher mean PA.

\section{Sleep Quality Variability}

Table 2 summarizes the results from models that examined the relationship between sleep quality and PA. In both samples, people who had higher mean sleep quality across the week also reported higher mean PA. People who had higher mean sleep quality also reported greater PA variability, but in the student sample only. In both samples, people with more variable sleep quality exhibited greater fluctuations in PA throughout the week (Fig. 1). Contrary to our expectations, more variable sleep quality was also associated with higher mean PA across both samples (Supplementary Figure S1).

\section{Sleep Duration Variability}

Table 3 and Supplementary Figure S2 summarize the results from models that examined the relationship between sleep duration and PA. In the community sample only, people with longer mean sleep duration tended to have higher mean PA, whereas more variable sleep duration was associated with lower mean PA. Additionally, participants from the community sample who had greater variability in sleep duration also exhibited more fluctuations in PA across the week. However, there were no significant relationships between sleep duration and PA in the student sample.

\section{Variability in Sleep Timing and WASO}

Supplementary Tables S3, S4, and S5 summarize the results from models that examined the associations of variability in bedtime, rise-time, and WASO with PA, respectively. After controlling for NA variability, there were no significant relationships between bedtime and PA in either sample (Table S3). In the community sample only, people who had earlier rise-times on average also had higher mean PA (Table S4). However, rise-time variability was not associated with mean PA nor PA variability in either sample. There were no significant relationships between WASO and PA in either sample (Table S5).

\section{Discussion}

This study examined the associations of intraindividual variability in sleep dimensions with mean PA and PA variability in two adult samples. We hypothesized that people with more variable night-to-night sleep would be at risk for lower and more variable daily PA across a week. Our hypothesis was partially supported, such that greater variability in sleep quality was associated with greater fluctuations in PA throughout the week in both samples. Additionally, individuals with more variable sleep duration also had more variable and lower PA in the community sample. Contrary to our expectations, in both samples, people with more variable sleep quality had higher levels of PA on average, and there were no significant associations between variability in sleep timing nor WASO with PA.

\section{Sleep Variability, PA Mean, and PA Variability}

Although several studies have shown that people with more variable PA tend to have shorter and less efficient sleep on average (e.g., Leger et al., 2019; Ong et al., 2013), existing research on sleep variability and mental health has predominantly focused on linkages with psychological distress (e.g., perceived stress, depressive symptoms; Bei et al., 2016; Veeramachaneni et al., 2019). Our study builds on and extends this literature on sleep variability by focusing on positive aspects of well-being, namely PA in daily life. People who have more sleep disturbances might subsequently have worse emotional functioning through REM sleep-related mechanisms, which help unlink emotion from prior affective experiences and recalibrate salience signalling in the brain (Goldstein \& Walker, 2014). Based on the framework described by Palmer and Alfano (2017), patterns of inconsistent sleep may influence various stages of the emotion generation and regulation process. For example, individuals with more variable sleep quality may be more likely to select themselves into situations (via impaired 


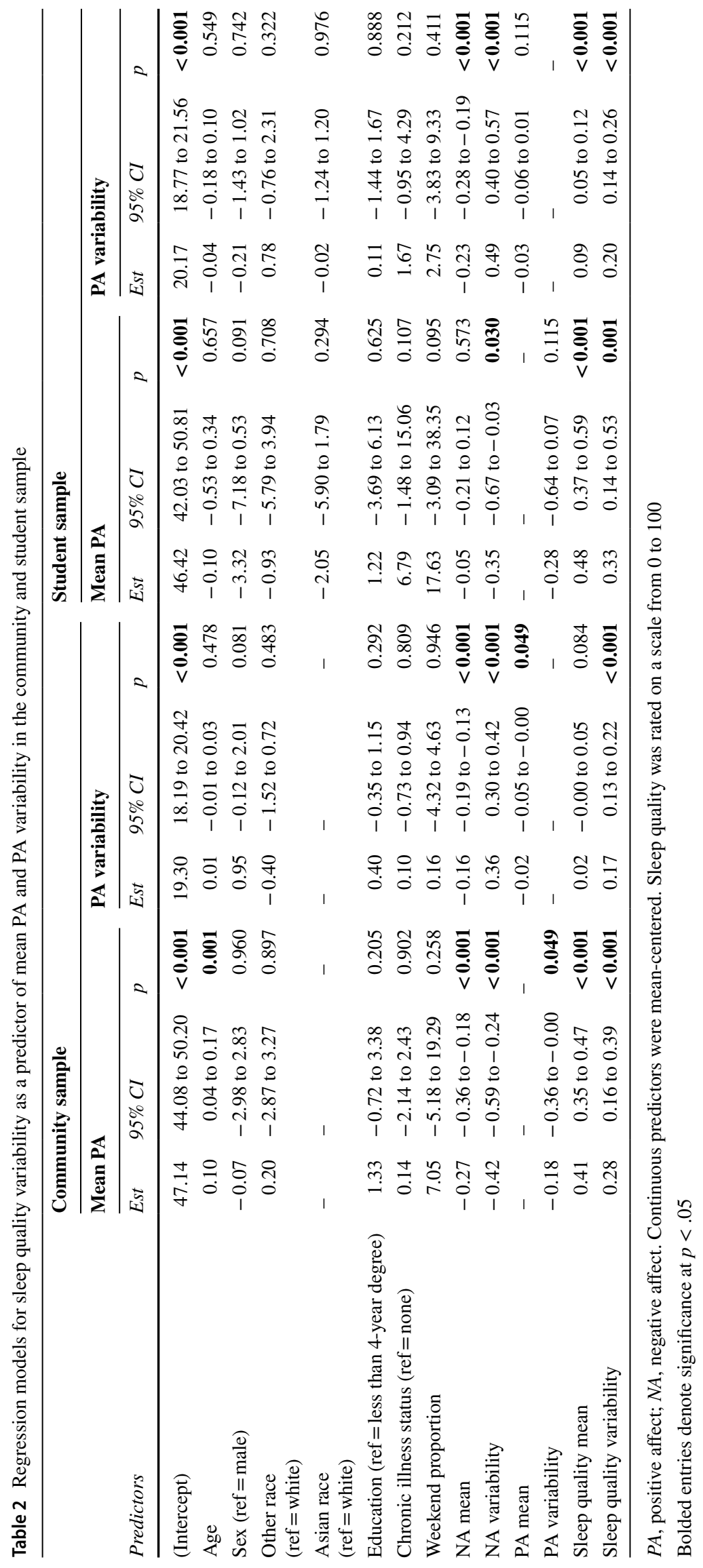




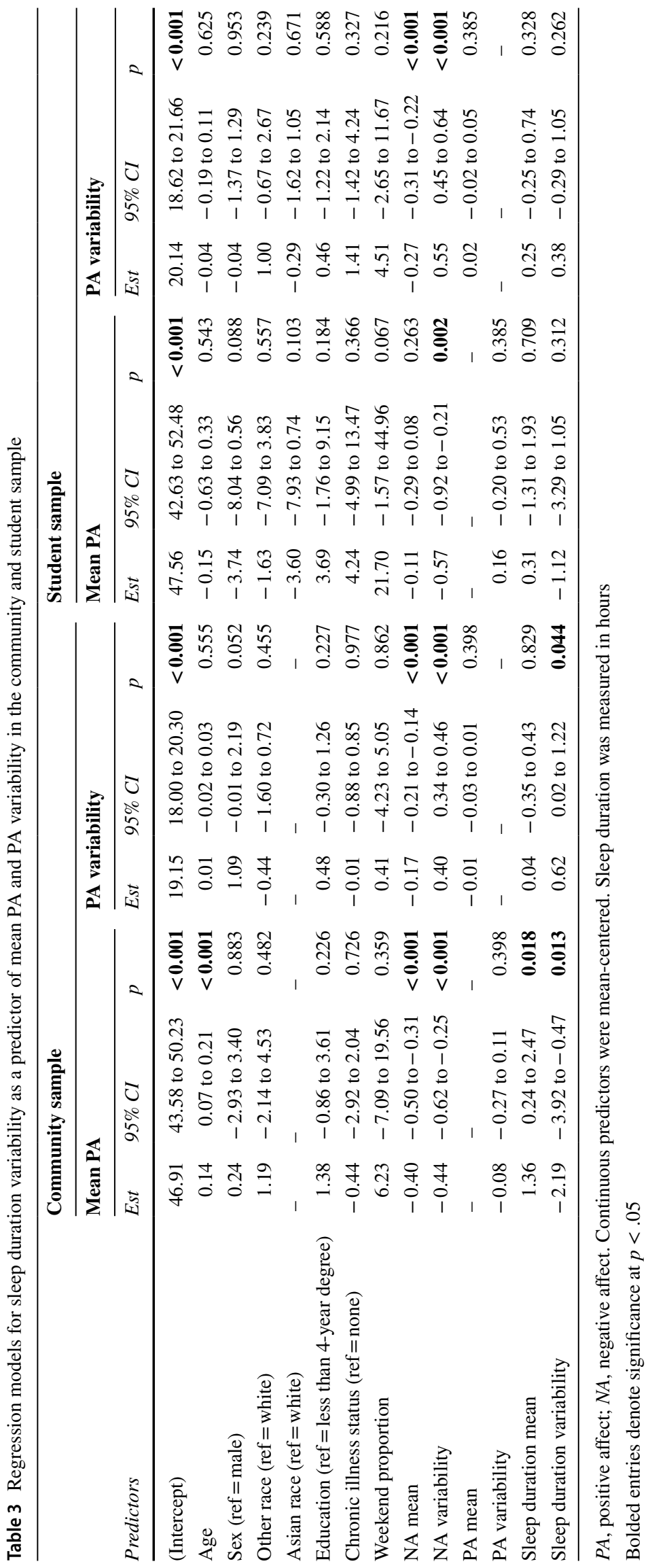




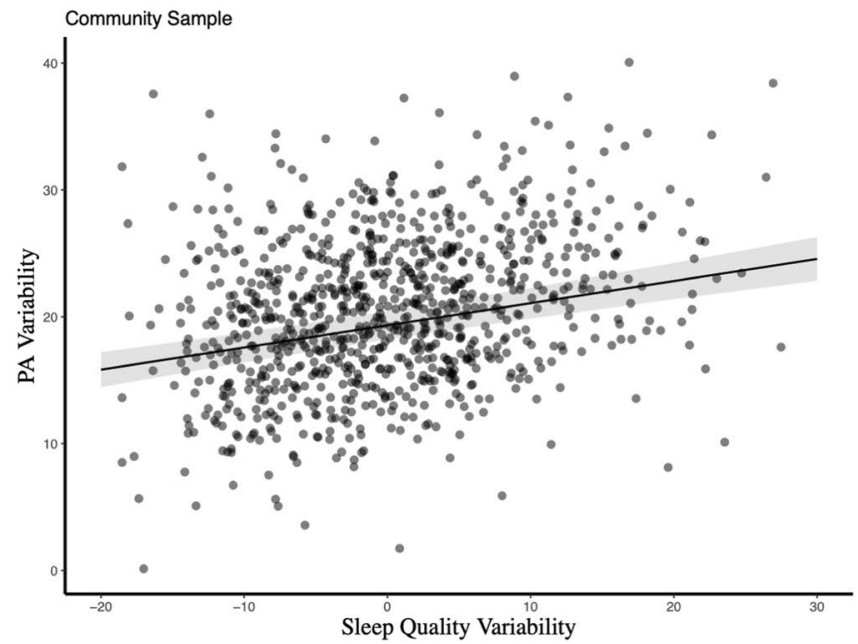

Fig. 1 Sleep quality variability and PA variability in the community and student samples. The positive linear association between sleep quality variability and PA variability was significant in both the community (left panel) and student (right panel) samples, even after

decision-making and impulse control; Womack et al., 2013) that then result in greater fluctuations in PA, or perhaps have greater affective reactivity to emotion-eliciting events (Sin et al., 2020). It is also possible that inconsistent sleep across consecutive nights disrupts one's circadian rhythm, which could then influence PA given previous research which indicates that daily variation in PA is partially regulated by endogenous mechanisms (Murray et al., 2002). However, we cannot rule out the possibility of a third variable (e.g., substance use or post-traumatic stress disorder, for which we did not have data) that could be linked to both sleep variability and PA dynamics.

Although there are several possible explanations as to why greater sleep quality variability was, unexpectedly, associated with higher mean PA, the exact mechanisms are unclear. On the one hand, it may be that variability in sleep quality reflects responsiveness to the environment, such that individuals with greater variability in sleep experience more social interactions or positive events, compared to individuals with less variable sleep quality. Indeed, the measure of PA used in this study includes items ("close to others" and "like you belong") that reflect feelings of social connection and possibly interpersonal interactions that may have occurred that day. Another possibility is that people with greater variability in sleep quality have more responsibilities that could impair their sleep, yet promote PA. For instance, people with more daily stressors tend to have better psychological well-being because they are more likely to be educated, employed, engaged in volunteering, and have family responsibilities (Almeida, 2005). However, although we have conceptualized sleep variability as a driver of affective dynamics, it is also possible that people who are happier

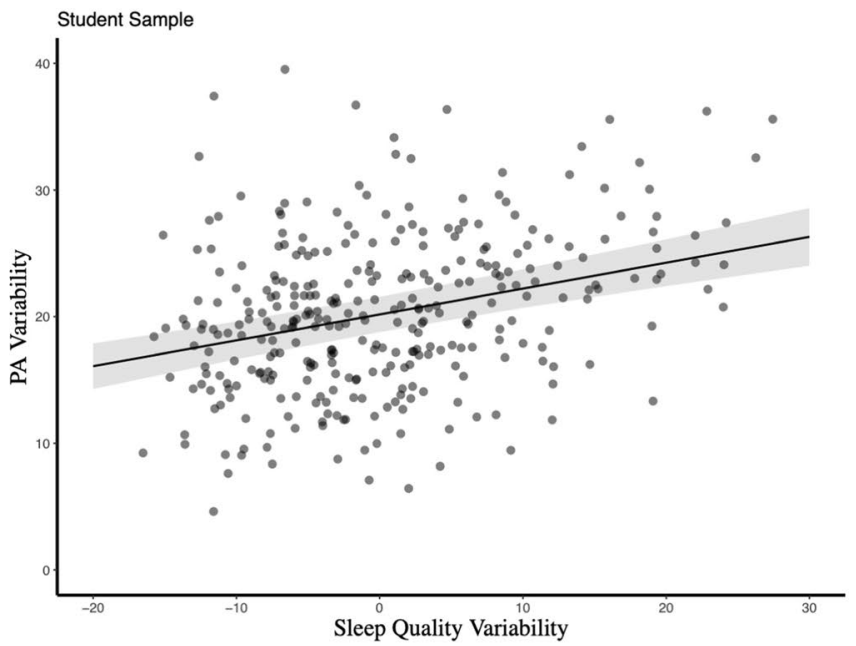

controlling for mean PA, mean sleep quality, IIV and mean NA, and demographic factors. Sleep quality variability was centered on the grand mean (mean $=18.51, S D=8.46$ for the community sample; mean $=17.37, S D=9.12$ for the student sample)

tend to experience more positive events and/or are more emotionally responsive to their environments, which may then result in irregular sleep patterns.

\section{Community Sample Versus Student Sample}

Although we did not have any hypotheses regarding differences between the community versus student populations, our findings were not altogether consistent between the two samples. The between-person association of sleep duration variability with lower mean PA, and of sleep duration variability with greater PA variability, was observed only in the community sample and not in the university student sample. To our knowledge, this is the first study examining sleep and PA in student as well as non-student samples, but the pattern we observed in the students (i.e., mean and variability in sleep quality, but not duration, were related to PA) was in line with previous literature on the sleep-PA relationship in university students. For example, Kalmbach et al. (2014) found that only higher sleep quality, but not total sleep time nor sleep onset latency, predicted higher next-day PA in women university students. Similarly, Pressman et al. (2017) found that trait PA was related to higher sleep quality and efficiency, but not duration, during the week of a major exam. This is particularly relevant given that our data were collected when students were preparing for final exams amid the transition to remote learning during the pandemic. A possible explanation for the less robust relationship between sleep duration and PA in our university student sample versus community sample is that external factors may be more important for students' sleep duration. As Pressman et al. (2017) point out, students frequently 
sacrifice sleep for school, work, or social commitments. Furthermore, younger adults may be more resilient to poorer quality sleep and as such, sleep may not be as closely tied to affect in students. Indeed, only sleep quality was correlated with PA in students, in contrast to many sleep measures in the community sample.

\section{Limitations and Future Directions}

Limitations of the current study should be considered when interpreting its findings. First, we conceptualized variability in sleep as the driver of PA experiences, but we are unable to draw conclusions about directionality or causation due to the cross-sectional and correlational study design. Second, because data were collected entirely online, this study relied on self-reported sleep and affect. Studies examining the relationship between sleep and affect or stress using self-report (diary), inferred (actigraphy), and/or objective (electroencephalography) measures have sometimes found differential associations depending on the measure used (Slavish et al., 2020; McCrae et al., 2008; Messman et al., 2021). These different measures reflect partially distinct domains of sleep, which perhaps explains why subjective sleep quality, which captures one's holistic appraisal of sleep and can only be measured via self-report, had the most consistent associations with PA in our study. However, other research has reported fairly consistent links between daytime stress and sleep using both self-reported and actigraphic sleep assessments (Yap et al., 2020). Thus, the use of subjective versus objective sleep measurement when studying affect is far from fully understood and future research should take a multimethod approach for sleep assessment when possible (Slavish et al., 2020). Third, this study was conducted during the COVID-19 pandemic, and the patterns of night-to-night sleep and daily affect may not generalize to periods of lower stress. However, this was a unique opportunity to examine sleep and PA during a time of heightened disruption in daily life. Because this was a period of high stress and uncertainty, it is possible that people who were coping relatively well were more likely to participate in a daily diary study. Therefore, this dataset may not have captured individuals with the most disrupted sleep and affect. Finally, the community sample was recruited online and was primarily white, female, and well-educated. These factors related to the context and the samples could limit the generalizability of findings.

Future work could extend the current findings by investigating outstanding questions about the nature of variability in sleep and PA. First, it is possible that more variable sleep is associated with affective lability in general (i.e., both PA and NA variability). Much remains unknown about whether people with greater night-to-night variability in sleep might be unable to sustain their PA in particular or perhaps swing between positive and negative emotions. Further work is also needed to better contextualize variability in sleep and PA within one's environment and to determine when variability might reflect adaptive versus maladaptive responses to external events or when variability reflects intrinsic fluctuations that are not entirely due to external circumstances (e.g., Liu et al., 2018). For some individuals, fluctuations in sleep and affect may primarily indicate adaptive responsiveness to environmental demands, whereas variability may reflect oversensitivity to the environment or poorer self-regulation for others. Answering these questions may help identify individuals who are more emotionally vulnerable to poorer or shorter sleep and at greater risk for developing future health conditions (Sin et al., 2021; Vigoureux et al., 2020).

\section{Conclusion}

To our knowledge, this study was among the first to investigate the relationship between intraindividual variability in multiple indicators of sleep health (quality, duration, WASO, bedtime, and rise-time) with mean level and variability in PA. Based on parallel analyses in community-based adults and university students, our findings suggest that inconsistency in sleep patterns is linked to both better and worse psychological well-being. Specifically, people with more variable sleep quality had more variable and higher mean PA than those with less-variable sleep quality, whereas individual differences in sleep duration variability predicted more variable and lower mean PA. Future work should investigate potential mechanisms - such as neurobiological pathways and responsiveness to environmental contexts - that underlie the links between patterns of inconsistent sleep and affective dynamics.

\section{Additional Information}

Funding This work was supported by the Social Sciences and Humanities Research Council of Canada (430-2019-00387 to NLS, 435-20161350 to AD) and the Michael Smith Foundation for Health Research (SCH-2020-0590 to NLS).

Data Availabity Data, code, and materials are available on the Open Science Framework website at https://osf.io/wq5xh/?view_only=062ae 85dafcf4fafb16658128a1a5191.

Ethics Approval Study procedures were approved by the Behavioural Research Ethics Board at the University of British Columbia (\# H1904073).

Conflict of Interest Dr. Slavish reports industry grant funding from Canopy Growth Corporation that is outside the scope of the current work. The other authors have no conflicts of interest to report.

Supplementary Information The online version contains supplementary material available at https://doi.org/10.1007/s42761-021-00082-6. 


\section{References}

Almeida, D. M. (2005). Resilience and vulnerability to daily stressors assessed via diary methods. Current Directions in Psychological Science, 14, 64-68.

Bei, B., Wiley, J. F., Trinder, J., \& Manber, R. (2016). Beyond the mean: A systematic review on the correlates of daily intraindividual variability of sleep/wake patterns. Sleep Medicine Reviews, $28,108-124$

Boehm, J. K., \& Kubzansky, L. D. (2012). The heart's content: The association between positive psychological well-being and cardiovascular health. Psychological Bulletin, 138, 655.

Bouwmans, M. E., Bos, E. H., Hoenders, H. R., Oldehinkel, A. J., \& de Jonge, P. (2017). Sleep quality predicts positive and negative affect but not vice versa. An electronic diary study in depressed and healthy individuals. Journal of Affective Disorders, 207, 260-267.

Buysse, D. J. (2014). Sleep health: Can we define it? Does it matter? Sleep, 37, 9-17.

Carney, C. E., Buysse, D. J., Ancoli-Israel, S., Edinger, J. D., Krystal, A. D., Lichstein, K. L., \& Morin, C. M. (2012). The consensus sleep diary: Standardizing prospective sleep self-monitoring. Sleep, 35, 287-302.

Chida, Y., \& Steptoe, A. (2008). Positive psychological well-being and mortality: A quantitative review of prospective observational studies. Psychosomatic Medicine, 70, 741-756.

Chan, D. K., Zhang, X., Fung, H. H., \& Hagger, M. S. (2016). Affect, affective variability, and physical health: Results from a population-based investigation in China. International Journal of Behavioral Medicine, 23, 438-446.

Charles, S. T., Mogle, J., Leger, K. A., \& Almeida, D. M. (2019). Age and the factor structure of emotional experience in adulthood. The Journals of Gerontology: Series B, 74, 419-429.

Cranford, J. A., Shrout, P. E., Iida, M., Rafaeli, E., Yip, T., \& Bolger, N. (2006). A procedure for evaluating sensitivity to withinperson change: Can mood measures in diary studies detect change reliably? Personality and Social Psychology Bulletin, 32, 917-929.

Eid, M., \& Diener, E. (1999). Intraindividual variability in affect: Reliability, validity, and personality correlates. Journal of Personality and Social Psychology, 76, 662.

Finan, P. H., Quartana, P. J., Remeniuk, B., Garland, E. L., Rhudy, J. L., Hand, M., Irwin, M. R., \& Smith, M. T. (2017). Partial Sleep Deprivation Attenuates the Positive Affective System: Effects Across Multiple Measurement Modalities. Sleep, 40(1).

Goldstein, A. N., \& Walker, M. P. (2014). The role of sleep in emotional brain function. Annual Review of Clinical Psychology, 10, 679-708.

Gradisar, M., Wolfson, A. R., Harvey, A. G., Hale, L., Rosenberg, R., \& Czeisler, C. A. (2013). The sleep and technology use of Americans: Findings from the National Sleep Foundation's 2011 Sleep in America poll. Journal of Clinical Sleep Medicine, 9, 1291-1299.

Gross, J. J. (1998). The emerging field of emotion regulation: An integrative review. Review of General Psychology, 2, 271-299.

Gruber, J., Kogan, A., Quoidbach, J., \& Mauss, I. B. (2013). Happiness is best kept stable: Positive emotion variability is associated with poorer psychological health. Emotion, 13, 1.

Hardy, J., \& Segerstrom, S. C. (2017). Intra-individual variability and psychological flexibility: Affect and health in a National US sample. Journal of Research in Personality, 69, 13-21.

Human, L. J., Whillans, A. V., Hoppmann, C. A., Klumb, P., Dickerson, S. S., \& Dunn, E. W. (2015). Finding the middle ground: Curvilinear associations between positive affect variability and daily cortisol profiles. Emotion, 15, 705.
Houben, M., Van Den Noortgate, W., \& Kuppens, P. (2015). The relation between short-term emotion dynamics and psychological well-being: A meta-analysis. Psychological Bulletin, 141, 901.

Jenkins, B. N., Hunter, J. F., Cross, M. P., Acevedo, A. M., \& Pressman, S. D. (2018). When is affect variability bad for health? The association between affect variability and immune response to the influenza vaccination. Journal of Psychosomatic Research, 104, 41-47.

Jones, D. R., Smyth, J. M., Engeland, C. G., Sliwinski, M. J., Russell, M. A., Sin, N. L., ... \& Graham-Engeland, J. E. (2020). Affect variability and inflammatory markers in midlife adults. Health Psychology, 39; 655

Kahn, M., Sheppes, G., \& Sadeh, A. (2013). Sleep and emotions: Bidirectional links and underlying mechanisms. International Journal of Psychophysiology, 89, 218-228.

Kalmbach, D. A., Pillai, V., Roth, T., \& Drake, C. L. (2014). The interplay between daily affect and sleep: A 2-week study of young women. Journal of Sleep Research, 23, 636-645.

Leger, K. A., Charles, S. T., \& Fingerman, K. L. (2019). Affect variability and sleep: Emotional ups and downs are related to a poorer night's rest. Journal of psychosomatic research, 124, 109758.

Liu, Y., Bangerter, L. R., Rovine, M. J., Zarit, S. H., \& Almeida, D. M. (2018). Intrinsic emotional fluctuation in daily negative affect across adulthood. The Journals of Gerontology: Series B, 73 , $100-112$.

Lyubomirsky, S., King, L., \& Diener, E. (2005). The benefits of frequent positive affect: Does happiness lead to success? Psychological bulletin, 131, 803.

Maccari, L., Martella, D., Marotta, A., Sebastiani, M., Banaj, N., Fuentes, L. J., \& Casagrande, M. (2014). Effects of sleep loss on emotion recognition: A dissociation between face and word stimuli. Experimental Brain Research, 232, 3147-3157.

McCrae, C. S., McNamara, J. P., Rowe, M. A., Dzierzewski, J. M., Dirk, J., Marsiske, M., \& Craggs, J. G. (2008). Sleep and affect in older adults: Using multilevel modeling to examine daily associations. Journal of Sleep Research, 17, 42-53.

Messman, B. A., Slavish, D. C., Dietch, J. R., Jenkins, B. N., ten Brink, M., \& Taylor, D. J. (2021). Associations between daily affect and sleep vary by sleep assessment type: What can ambulatory EEG add to the picture? Sleep Health, 7(2), 219-228.

Mezick, E. J., Matthews, K. A., Hall, M., Kamarck, T. W., Buysse, D. J., Owens, J. F., \& Reis, S. E. (2009). Intra-individual variability in sleep duration and fragmentation: Associations with stress. Psychoneuroendocrinology, 34, 1346-1354.

Murray, G., Allen, N. B., \& Trinder, J. (2002). Mood and the circadian system: Investigation of a circadian component in positive affect. Chronobiology International, 19, 1151-1169.

Ong, A. D., Exner-Cortens, D., Riffin, C., Steptoe, A., Zautra, A., \& Almeida, D. M. (2013). Linking stable and dynamic features of positive affect to sleep. Annals of Behavioral Medicine, 46, 52-61.

Ong, A. D., Kim, S., Young, S., \& Steptoe, A. (2017). Positive affect and sleep: A systematic review. Sleep Medicine Reviews, 35, 21-32.

Ong, A. D., \& Ram, N. (2017). Fragile and enduring positive affect: Implications for adaptive aging. Gerontology, 63, 263-269.

Ong, A. D., \& Steptoe, A. (2020). Association of positive affect instability with all-cause mortality in older adults in England. JAMA Network Open, 3, e207725-e207725.

Palmer, C. A., \& Alfano, C. A. (2017). Sleep and emotion regulation: An organizing, integrative review. Sleep Medicine Reviews, 31, 6-16.

Prati, G., \& Mancini, A. D. (2021). The psychological impact of COVID-19 pandemic lockdowns: A review and meta-analysis of longitudinal studies and natural experiments. Psychological medicine, $1-11$ 
Pressman, S. D., Jenkins, B. N., Kraft-Feil, T. L., Rasmussen, H., \& Scheier, M. F. (2017). The whole is not the sum of its parts: Specific types of positive affect influence sleep differentially. Emotion, 17(5), 778

Pressman, S. D., Jenkins, B. N., \& Moskowitz, J. T. (2019). Positive affect and health: What do we know and where next should we go? Annual Review of Psychology, 70, 627-650.

R Core Team (2020). R: A language and environment for statistical computing (Version 4.0.2) R Foundation for Statistical Computing, Vienna, Austria. URL https://www.R-project.org/

Rezaei, N., \& Grandner, M. A. (2021). Changes in sleep duration, timing, and variability during the COVID-19 pandemic: Large-scale Fitbit data from 6 major US cities. Sleep Health, 7(3), 303-313.

Robinson, E., Sutin, A. R., Daly, M., \& Jones, A. (2021). A systematic review and meta-analysis of longitudinal cohort studies comparing mental health before versus during the COVID-19 pandemic in 2020. Journal of Affective Disorders, S0165-0327(21)01057-0.

Sin, N. L., Almeida, D. M., Crain, T. L., Kossek, E. E., Berkman, L. F., \& Buxton, O. M. (2017). Bidirectional, temporal associations of sleep with positive events, affect, and stressors in daily life across a week. Annals of Behavioral Medicine, 51, 402-415.

Sin, N. L., Klaiber, P., Wen, J. H., \& DeLongis, A. (2021). Helping amid the pandemic: Daily affective and social implications of COVID-19-related prosocial activities. The Gerontologist, 61, $59-70$.

Sin, N. L., Rush, J., Buxton, O. M., \& Almeida, D. M. (2021). Emotional Vulnerability to Short Sleep Predicts Increases in Chronic Health Conditions Across 8 Years. Annals of Behavioral Medicine, kaab018.

Sin, N. L., Wen, J. H., Klaiber, P., Buxton, O. M., \& Almeida, D. M. (2020). Sleep duration and affective reactivity to stressors and positive events in daily life. Health Psychology, 39(12), $1078-1088$.

Slavish, D. C., Asbee, J., Veeramachaneni, K., Messman, B. A., Scott, B., Sin, N. L., Taylor, D. J., \& Dietch, J. R. (2021). The Cycle of Daily Stress and Sleep: Sleep Measurement Matters. Annals of Behavioral Medicine, 55(5), 413-423.
Slavish, D. C., Taylor, D. J., Dietch, J. R., Wardle-Pinkston, S., Messman, B., Ruggero, C. J., ... \& Kelly, K. (2020). Intraindividual variability in sleep and levels of systemic inflammation in nurses. Psychosomatic Medicine, 82, 678-688

Slavish, D. C., Taylor, D. J., \& Lichstein, K. L. (2019). Intraindividual variability in sleep and comorbid medical and mental health conditions. Sleep, 42, zsz052

Steptoe, A., Wardle, J., \& Marmot, M. (2005). Positive affect and health-related neuroendocrine, cardiovascular, and inflammatory processes. Proceedings of the National Academy of Sciences, 102, 6508-6512.

Veeramachaneni, K., Slavish, D. C., Dietch, J. R., Kelly, K., \& Taylor, D. J. (2019). Intraindividual variability in sleep and perceived stress in young adults. Sleep Health, 5, 572-579.

Vigoureux, T. F., Lee, S., Buxton, O. M., \& Almeida, D. M. (2020). Stressor reactivity to insufficient sleep and its association with body mass index in middle-aged workers. Journal of sleep research, 29, e12955

Wen, J. H., Klaiber, P., DeLongis, A., Slavish, D. C., \& Sin, N. L. (in press). Day-to-day associations of sleep with health and wellbeing amid the COVID-19 pandemic in North America. Sleep Health.

Womack, S. D., Hook, J. N., Reyna, S. H., \& Ramos, M. (2013). Sleep loss and risk-taking behavior: A review of the literature. Behavioral Sleep Medicine, 11, 343-359.

Yap, Y., Slavish, D. C., Taylor, D. J., Bei, B., \& Wiley, J. F. (2020). Bidirectional relations between stress and self-reported and actigraphy-assessed sleep: A daily intensive longitudinal study. Sleep, 43, zsz250

Zheng, J., Morstead, T., Sin, N., Klaiber, P., Umberson, D., Kamble, S., \& DeLongis, A. (2021). Psychological distress in North America during COVID-19: The role of pandemic-related stressors. Social Science \& Medicine, 270, 113687

Zohar, D., Tzischinsky, O., Epstein, R., \& Lavie, P. (2005). The effects of sleep loss on medical residents' emotional reactions to work events: A cognitive-energy model. Sleep, 28, 47-54. 